Escuela de Kinesiología, Facultad de Salud, Universidad Santo Tomás, Santiago, Chile. angeniero Biomédico, MSc (c). ${ }^{\mathrm{b} K i n e s i o ́ l o g o, ~ M S c ~(c) . ~}$

Ambos autores contribuyeron de igual forma para este trabajo.

Apoyo Financiero: Universidad Santo Tomás, proyecto de investigación código TAS 0000015822

Recibido el 23 de marzo de 2015, aceptado el 27 de octubre de

2015.

Correspondencia a: Ricardo A. Ulloa J. Teléfono: +56224717674 ricardoulloa@santotomas.cl

\section{Cambios asociados al envejecimiento normal en los parámetros angulares de la marcha a una velocidad controlada}

\author{
MAILYN J. CALDERÓN D. ${ }^{\text {a }, ~ R I C A R D O ~ A . ~ U L L O A ~ J . ~}{ }^{\text {}}$
}

\section{Changes associated with aging in angular kinematic parameters during a controlled speed walk}

Background: During normal aging, a reduction in walking speed and changes in stability are observed. Aim: To characterize and compare gait kinematic parameters of young (YA) and older adults (OA) at a controlled speed. Material and Methods: Gait angular kinematic parameters were analyzed in $O A$ aged over 60 years and YA aged between 20 and 30 years. For data capture, six VICON Bonita cameras were used. Recording were made at a gait speed of $1.2 \mathrm{~m} / \mathrm{s}$. Kinematic data were normalized to $100 \%$ of the gait cycle using the Polygon 4.1 software. Time windows representing the phases of gait were created for analysis. Results: At the hip-pelvis level, OA reduce their pelvic obliquity motion and increase hip abduction. At the knee level OA reduce movements in the frontal plane during the plantar support and half swing phases. At the ankle level $O A$ reduce their maximum ranges of plantar flexion, during the toe-off and final swing phases. Conclusions: At a controlled speed, kinematic changes observed in $O A$ allow an increase in body support base to maintain gait stability.

(Rev Med Chile 2016; 144: 74-82)

Key words: Aging; Gait; Movement.

\section{L} a marcha normal es el producto final de un sistema neuromusculoesquelético sano, la las grandes articulaciones del cuerpo. Un ciclo de marcha ha sido definido como el tiempo desde el contacto de talón hasta el siguiente contacto de talón ipsilateral ${ }^{1}$. Para que la marcha sea eficaz y económica en términos energéticos, distintos sistemas como el nervioso, osteomuscular, visual, somatosensorial, vestibular y cognitivo entre otros, deben interactuar correctamente ${ }^{2}$. Con respecto a esto, se ha descrito que durante el envejecimiento normal existe una pérdida progresiva de la funcionalidad de los sistemas que contribuyen al control postural ${ }^{3-5}$, así como cambios a nivel musculoesquelético que afectan su función, tales como la sarcopenia ${ }^{6-9}$. Todos estos factores conlle- van cambios a nivel de tareas funcionales como la marcha ${ }^{8,10}$, generando inestabilidad y aumentando el riesgo de sufrir caídas ${ }^{8}$.

Uno de los factores que cambian en la marcha producto del envejecimiento es la velocidad a la que ésta se realiza ${ }^{11}$. Estudios anteriores han demostrando que los adultos mayores (AM) reducen su velocidad, aumentando la variabilidad angular de esta tarea y generando cambios en la estabilidad dinámica general ${ }^{11}$. Marchas a menores velocidades son comunes en los AM y de acuerdo a algunos estudios la reducción de esta sería un predictor del riesgo de caídas ${ }^{12}$. La reducción de la velocidad observada durante la marcha en los AM buscaría mantener la estabilidad dinámica, ya que a mayores velocidades se han observado patrones motores de mayor inestabilidad durante 
el envejecimiento ${ }^{12-13}$. También se ha determinado que la inestabilidad durante la marcha ocurre principalmente en dirección medio-lateral ${ }^{14}$, asociándose a un mayor riesgo de caídas en AM, precisamente cuando estos caminan a mayores velocidades ${ }^{15}$. Sin embargo, otros estudios sugieren que el incremento de la variabilidad angular durante la marcha sería el predictor de caídas y no las bajas velocidades ${ }^{14-16}$. Esto hace necesario conocer qué ocurre en la cinemática angular de la marcha cuando comparamos a AM y adultos jóvenes (AJ) a una velocidad controlada, ya que, de acuerdo a estudios previos, las diferencias en las características de la marcha se reducirían cuando la velocidad de ésta ha sido controlada ${ }^{17}$.

En la actualidad existe una gran cantidad de estudios concernientes a la variabilidad motora de la marcha y el envejecimiento en relación a parámetros cinemáticos. Sin embargo, los reportes específicos acerca de los mecanismos que contribuyen al envejecimiento y los cambios en los patrones de marcha han sido inconsistentes ${ }^{18,19}$.

El objetivo de este estudio fue comparar los desplazamientos angulares de tobillo, rodilla, cadera y pelvis durante la marcha en $\mathrm{AJ}$ y AM sanos a una velocidad controlada. El modelo propuesto nos permite determinar cómo se caracterizan los cambios en las estrategias cinemáticas angulares de la marcha producto del envejecimiento para toma de decisiones clínicas con una muestra chilena.

\section{Material y Métodos}

\section{Participantes}

Se realizó un estudio comparativo en dos grupos: $34 \mathrm{AJ}$ ( 20 a 30 años; 19 mujeres y 15 hombres; edad promedio $22,7 \pm 1,7$ años) y $38 \mathrm{AM}$ sanos (mayores de 60 años; 23 mujeres y 15 hombres; edad promedio 72,5 \pm 7,1 años). La muestra de AJ fue reclutada en la Universidad Santo Tomás (UST) sede de Santiago de Chile, y la de AM en la Asociación Cristiana de Jóvenes (YMCA), sede de Santiago, Chile. Cada sujeto fue sometido al test cognitivo Montreal Cognitive Assessment (MOCA) el cual mide deterioro cognitivo leve. Los criterios de exclusión fueron hiperlaxitud, sedentarismo, patologías visuales no tratadas, patologías músculo-esqueléticas diagnosticadas, patologías neurológicas centrales o periféricas, patologías sensoriales no corregidas, patologías degenerativas, obesidad o desnutrición, cáncer, trastornos del equilibrio, pie plano, uso de fármacos que afecten el control postural, MOCA $<26$ puntos y antecedentes de caídas en los últimos doce meses. Este estudio fue aprobado por el comité de ética de la UST. Todos los sujetos firmaron el consentimiento informado antes de su participación.

\section{Instrumentación y protocolo}

Los datos cinemáticos 3D fueron medidos a $100 \mathrm{~Hz}$ en la pierna dominante de los sujetos usando un sistema de 6 cámaras Vicon-Bonita (Vicon Motion System, Oxford, UK), con el modelo Plug-in gait, el cual utiliza marcadores reflectantes de $14 \mathrm{~mm}$ ubicados en los siguientes puntos: cabeza del segundo metatarsiano, maléolo externo, calcáneo, punto medio de la zona lateral de la pierna, cabeza de la fíbula, cóndilo femoral lateral, punto medio de la zona lateral del muslo, espinas ilíacas anterosuperiores y posterosuperiores. Este modelo permite realizar un análisis del comportamiento angular de cada articulación del tren inferior durante la marcha en cada plano de movimiento. Se utilizó un filtro pasa bajo Butterworth con una frecuencia de corte de $4 \mathrm{~Hz}$ para suavizar la trayectoria de los marcadores.

Los sujetos debieron caminar 5 veces por una pasarela de 8 metros de largo a una velocidad controlada de $1,2 \mathrm{~m} / \mathrm{s}$. Antes de la adquisición de los datos cada participante practicó por algunos minutos la marcha a esta velocidad.

\section{Procesamiento de los datos}

El ciclo de la marcha fue obtenido con el programa Nexus 2.0 (Vicon Motion System, Oxford, UK). El inicio se determinó por el primer contacto del talón con el suelo de la extremidad dominante, y el término con el cuarto contacto del talón ipsilateral, lo que equivale a 3 ciclos de la marcha.

Los datos fueron analizados en el programa Polygon 4.1 (Vicon Motion System, Oxford, UK), en el cual el tiempo de duración del ciclo de la marcha fue normalizado al $100 \%$, de los cuales la fase de apoyo representa aproximadamente $60 \%$ y la fase de balanceo el restante $40 \%$.

Se analizaron los desplazamientos angulares de las articulaciones de tobillo, rodilla, cadera y pelvis, en los planos sagital, frontal y transverso. 
Posteriormente se procedió a promediar las curvas angulares para cada articulación por plano de movimiento para ambos grupos.

Para comparar los datos por grupos etarios se crearon ventanas de tiempo correspondientes a las siguientes fases y porcentajes del ciclo de marcha: contacto de talón (0-3\%), apoyo plantar (3-20\%), apoyo medio (20-35\%), despegue de talón (35$50 \%)$, pre-balanceo (50-60\%) junto a balanceo inicial (60-73\%), balanceo medio (73-87\%), y balanceo final (87-100\%).

\section{Análisis estadístico}

Para evaluar las diferencias angulares de la marcha se utilizó la prueba de ANOVA de dos vías factorial con interacción, y una prueba $a$ posteriori de comparaciones múltiples con ajuste de Tukey-Kramer, y un valor alfa de 5\%. Estas pruebas fueron aplicadas a las ventanas de tiempo explicadas anteriormente. Las pruebas estadísticas fueron realizadas mediante el software SAS versión 9 (SAS Institute Inc.).

\section{Resultados}

La Tabla 1 muestra los resultados de la interacción en la variable grupo etario y la comparación múltiple de los rangos angulares máximos en cada fase de la marcha, en AJ y AM, correspondientes a las articulaciones de tobillo, rodilla, cadera y pelvis en los tres planos de movimiento para cada una de las ventanas temporales descritas anteriormente. Los resultados correspondientes a los desplazamientos angulares del ciclo de la marcha para cada articulación en el grupo de AJ y AM se observan en las Figuras 1 y 2 respectivamente. Las comparaciones entre las curvas cinemáticas angulares del ciclo de la marcha entre AM vs AJ, para las articulaciones de tobillo, rodilla, cadera y pelvis en cada plano de movimiento se observan en la Figura 3.

Se encontraron diferencias significativas $(\mathrm{p}<0,05)$ a nivel de cadera-pelvis en donde los AM reducen sus movimientos de oblicuidad pélvica y aumentan los de abducción de cadera. A nivel de rodilla, los AM reducen los movimientos en el plano frontal en las fases de apoyo plantar y balanceo medio. A nivel de tobillo, los AM disminuyen sus rangos máximos de flexión plantar en las fases de despegue de los dedos y balanceo final.

\section{Discusión}

\section{Articulación de tobillo}

Los movimientos del tobillo juegan un rol clave durante la locomoción humana. Durante la marcha, el tobillo permite atenuar los impactos de las fuerzas, ayuda a mantener el soporte y estabilidad de las extremidades inferiores y asiste en la propulsión anterior de la extremidad portante $^{19}$. Al comparar los movimientos de flexo-extensión de esta articulación, $\operatorname{Lim}^{17}$ encontró una disminución en los rangos máximos de flexión plantar en los AM. Estos resultados concuerdan con nuestros hallazgos, dado que encontramos diferencias en las fases de $\mathrm{PB} / \mathrm{BI}$ ( $\mathrm{p}$ $=0,0107)$ y en el BF $(p=0,0051)$. La reducción de la flexión plantar en los AM, sobre todo en la fase de $\mathrm{PB} / \mathrm{BI}$ puede deberse a una necesidad de aumentar el contacto suelo-pie, de tal forma de ampliar la base de soporte, lo que se traduce en un incremento de la estabilidad durante la marcha. Otro efecto que produce esta disminución angular es que los AM se caracterizan por generar una menor propulsión durante la marcha, lo que conlleva que en situaciones comunes a su vida diaria se ve afectada la velocidad de su marcha ${ }^{19-20}$. Por otra parte, la disminución de la flexión plantar en el BF buscaría posicionar el pie en el CT siguiente con una mayor base de apoyo, respondiendo a las necesidades de estabilidad que demandan los $\mathrm{AM}^{19-21}$.

Al comparar las curvas cinemáticas del tobillo en AM y AJ en todo el ciclo de marcha a velocidad controlada, ambos grupos presentan una gran variabilidad angular (gráficos superiores de Figuras 1 y 2) siendo siempre mayor en el grupo de AM. Por ejemplo, al observar los gráficos del plano transversal, se aprecia una variabilidad de hasta $70^{\circ}$ entre los valores máximos y mínimos en los AM entre las fases de apoyo medio y balanceo inicial versus una variabilidad de $60^{\circ}$ en los AJ. Este aumento en la variabilidad angular en los AM para algunos autores no sería un indicador de riesgo de caídas ${ }^{22-23}$. Sin embargo, para otros investigadores sí existiría un riesgo asociado a la variabilidad angular, cambio que era asociado a las variaciones de la velocidad de la marcha en $\mathrm{AM}^{14,16}$. Nosotros encontramos que al controlar la velocidad de la marcha siguen existiendo diferencias en la variabilidad angular entre ambos grupos. 


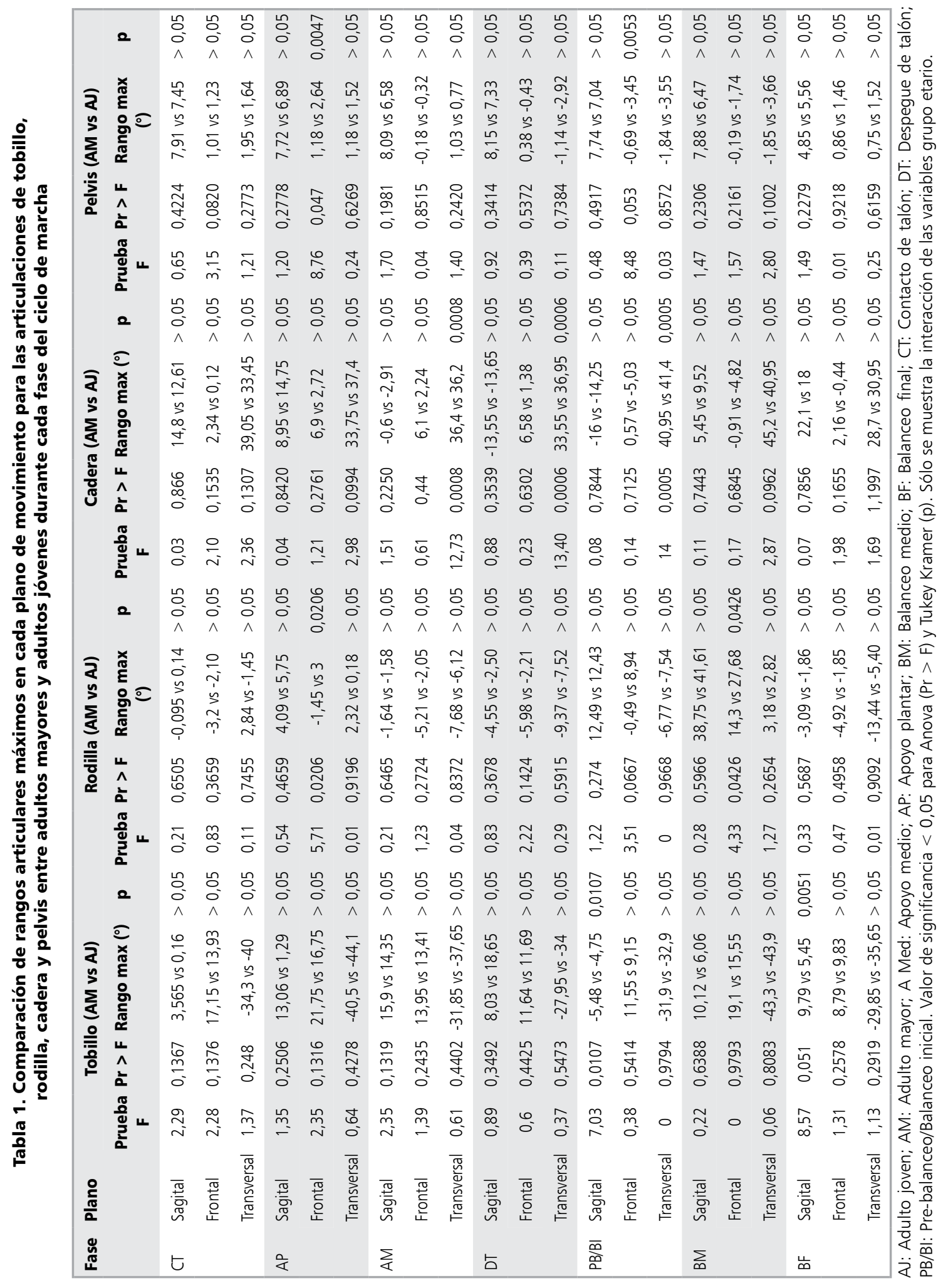



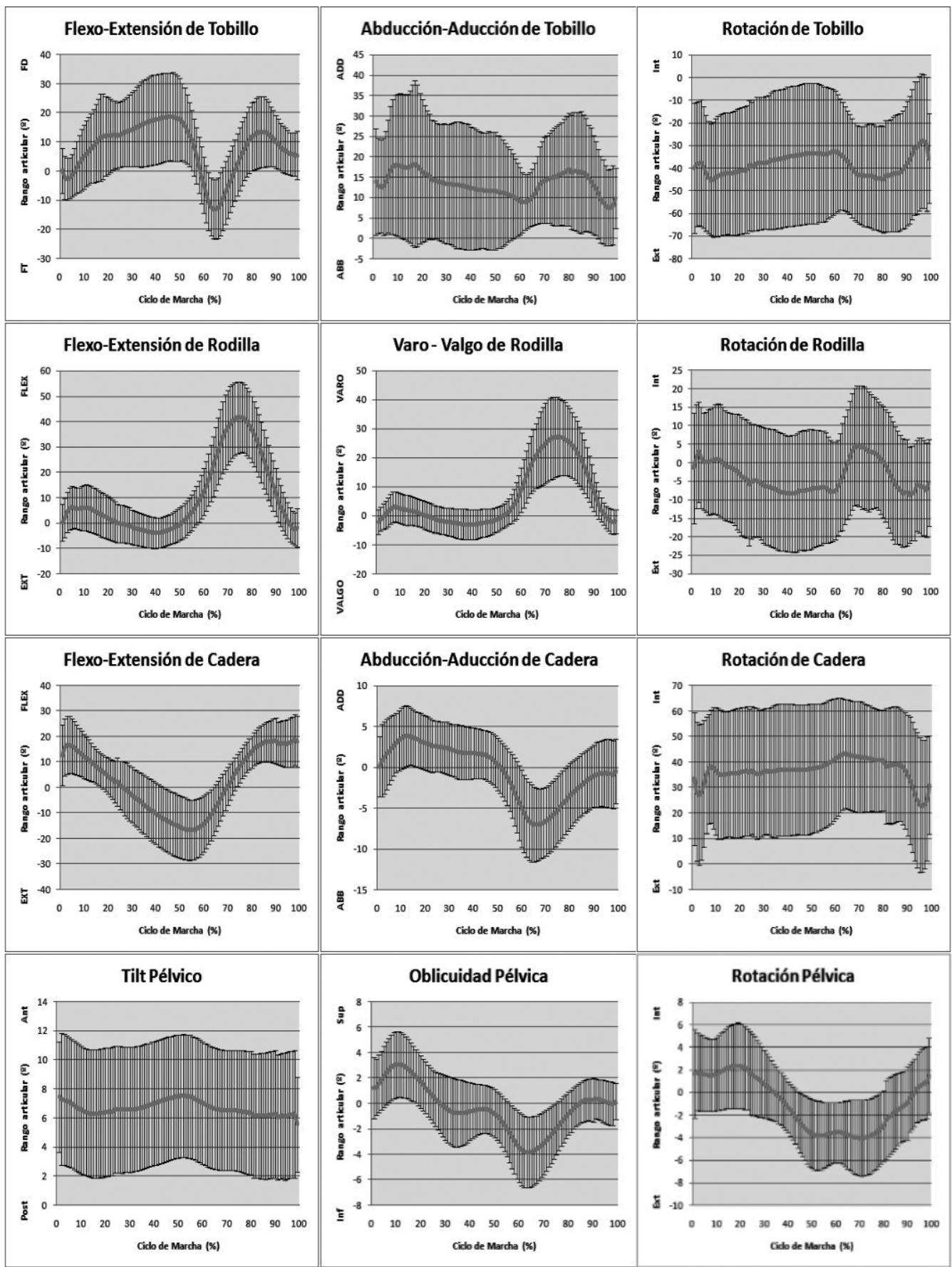

Figura 1. Desplazamientos angulares de tobillo, rodilla, cadera y pelvis (de superior a inferior) durante una marcha a velocidad controlada de 1,2 m/seg en el grupo de adultos jóvenes. En la primera columna se observan los movimientos en el plano sagital; en la segunda columna se observan los movimientos en el plano frontal; en la tercera columna se observan los movimientos en el plano transversal. La línea central negra representa los promedios de cada articulación, y las líneas verticales representan las desviaciones estándar. 

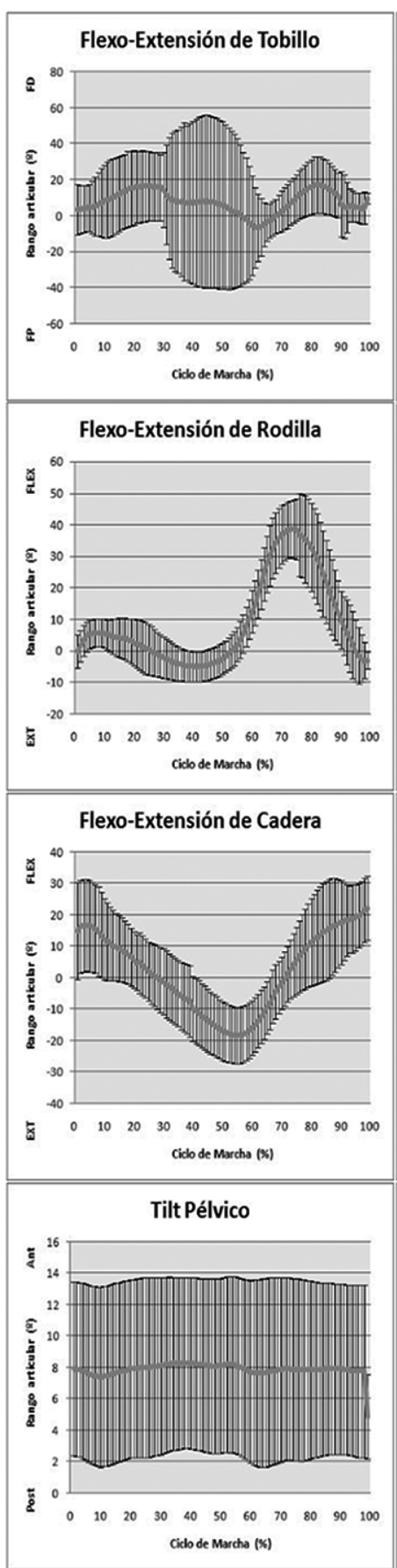
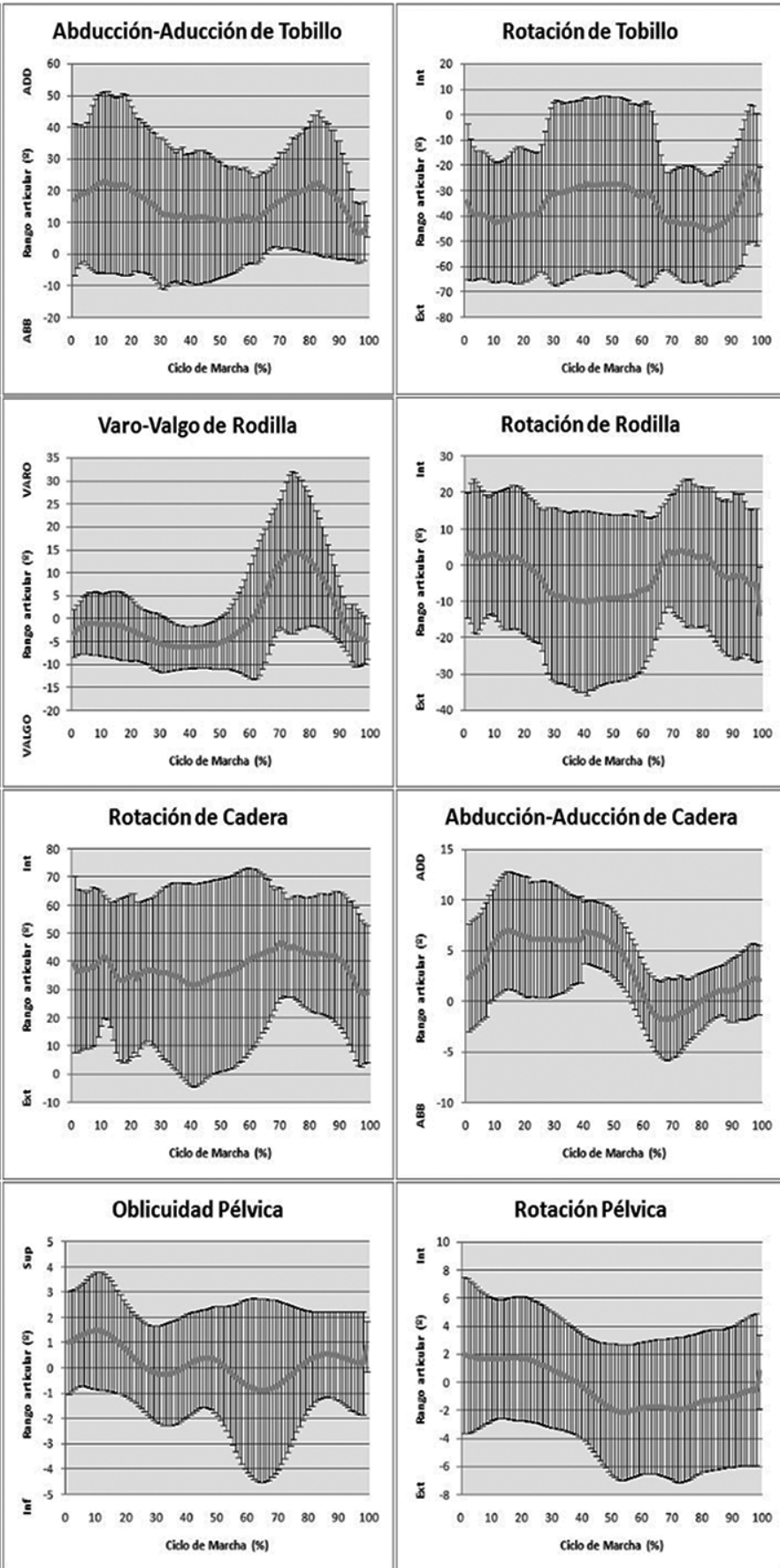
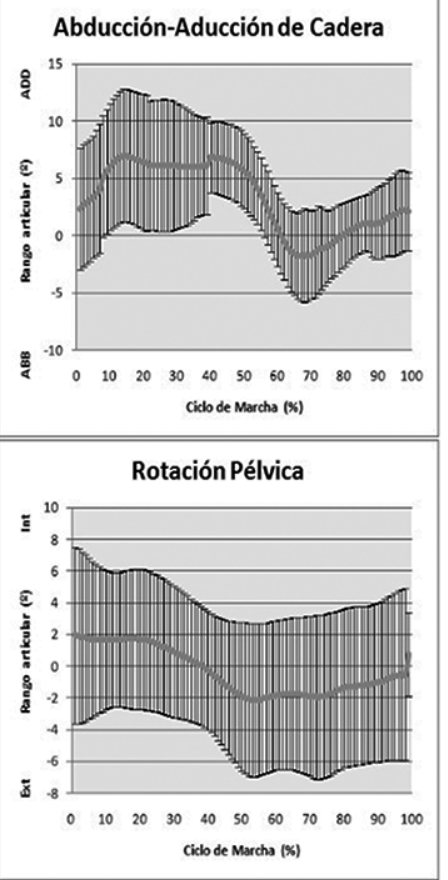

Figura 2. Desplazamientos angulares de tobillo, rodilla, cadera y pelvis (de superior a inferior) durante una marcha a velocidad controlada de 1,2 m/seg en el grupo de adultos mayores. En la primera columna se observan los movimientos en el plano sagital; en la segunda columna se observan los movimientos en el plano frontal; en la tercera columna se observan los movimientos en el plano transversal. La línea central negra representa los promedios de cada articulación, y las líneas verticales representan las desviaciones estándar. 


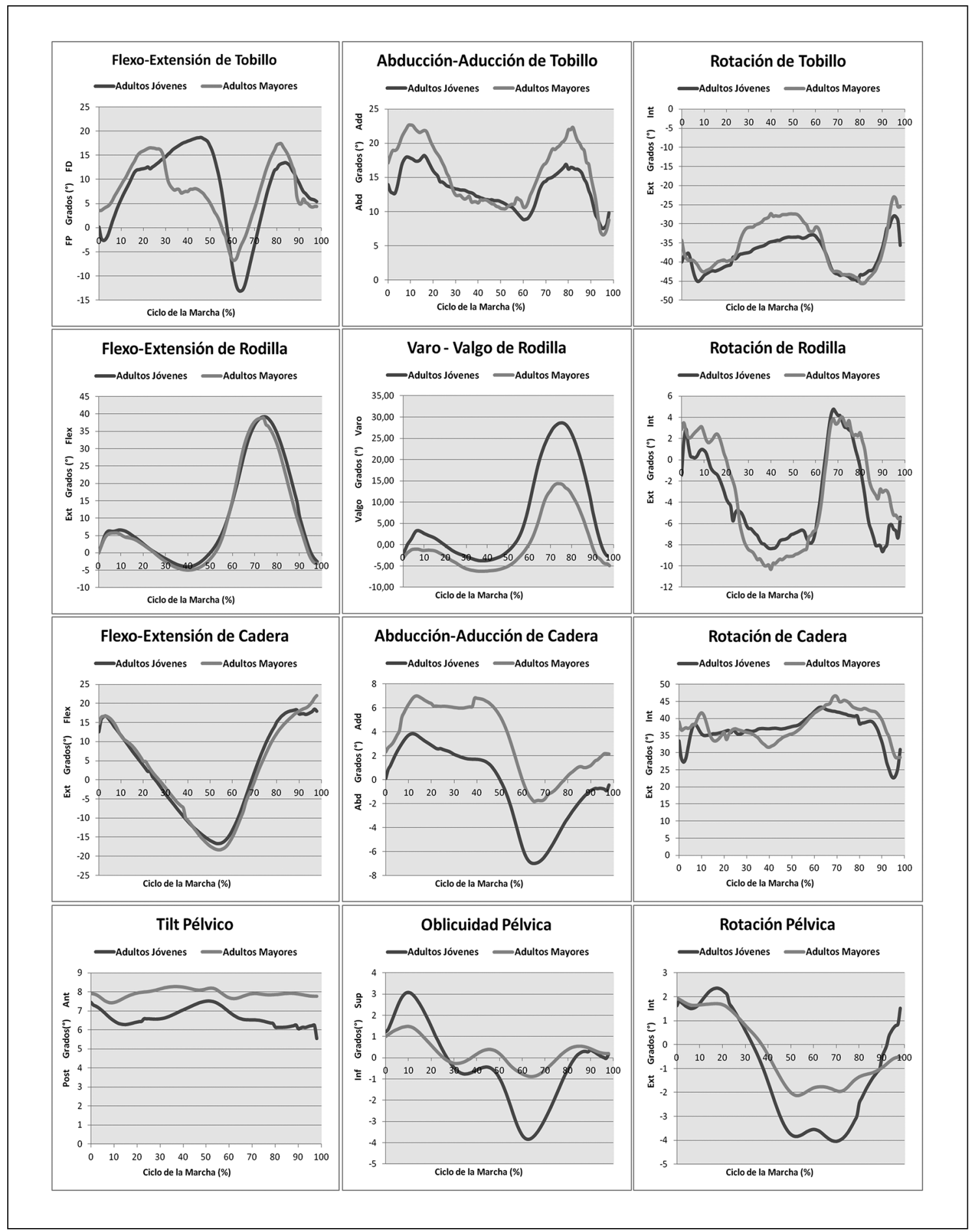

Figura 3. Comparación de desplazamientos angulares de tobillo, rodilla, cadera y pelvis (de superior a inferior) durante una marcha a velocidad controlada de 1,2 m/seg en los grupos de adultos mayores (línea negra) y adultos jóvenes (línea gris). En la primera columna se observan los movimientos en el plano sagital; en la segunda columna se observan los movimientos en el plano frontal; en la tercera columna se observan los movimientos en el plano transversal. 


\section{Articulación de la rodilla}

La rodilla es un complejo articular que se caracteriza por un amplio rango de movimiento en el plano sagital. Estos rangos son utilizados en la fase de apoyo de la marcha como un determinante en la estabilidad y, en la fase de balanceo como factor primario en el desplazamiento de las extremidades inferiores ${ }^{24}$.

Hasta el día de hoy, los resultados encontrados en la literatura son dispares, concordando que en general las diferencias en el movimiento de esta articulación son nulas o escasas ${ }^{2,15}$. Al estudiar el patrón de movimiento en el plano sagital en AM podemos darnos cuenta que mantiene los mismos rangos que en los AJ (Figura 3; p > 0,05).

$\mathrm{Al}$ contrastar los resultados de varo-valgo de esta articulación (Figura 3), encontramos que existen dos fases en las que hay una reducción del rango de movimiento en los AM: AP y BM $(\mathrm{p}=0,0206$ en ambas). Este cambio, al igual que en la articulación de la cadera, buscaría aumentar la estabilidad evitando una disminución del balance los AM.

Al comparar las curvas cinemáticas de la rodilla en AM y AJ en todo el ciclo de marcha a velocidad controlada, ambos grupos no presentan una gran variabilidad angular en el plano sagital, la cual si se observa en los planos frontal y transverso principalmente en el grupo de AM (Figuras 1 y 2). Si bien el grupo de AM redujo su rango de movimiento promedio en el plano frontal, a su vez aumentó la variabilidad angular en este, lo que de acuerdo a lo descrito por Schrager et al. sería un indicador de inestabilidad dinámica, aumentando el riesgo de sufrir una caída ${ }^{13}$.

\section{Articulaciones de cadera y pelvis}

Los movimientos de la cadera y la pelvis se realizan sincrónicamente, y su relación temporal no parece estar influenciada por el sexo ni por la edad $^{25}$. Estudios previos sugieren que en los AM el rango máximo de extensión de cadera es mayor y aumenta el tilt pélvico anterior ${ }^{1,17}$. Nuestros resultados (Figura 3) concuerdan con los datos reportadospor Lim en donde los AM alcanzan un máximo de extensión de cadera en la fase de $\mathrm{PB} /$ BI mayor a la de los AJ, y un tilt pélvico anterior superior al de los jóvenes durante todo el ciclo de la marcha ${ }^{19}$.

Una diferencia importante encontrada entre AM y AJ, es que los primeros reducen la oblicui- dad pélvica durante todo el ciclo de la marcha, alcanzando diferencias significativas en el apoyo plantar $(\mathrm{p}=0,0047)$ y en el despegue de los dedos $(p=0,0053)$. Este resultado indica que los AM al aumentar la inclinación anterior de la pelvis deben reducir los grados de libertad de los movimientos laterales de ésta, para así mantener la estabilidad dinámica que se requiere durante la marcha.

Un cambio esperado durante el envejecimiento es el que ocurre con los movimientos de abducción-aducción de cadera. Al observar el comportamiento angular, podemos apreciar que los AM aumentan su rango de abducción en más de $4^{\circ}$ durante todo el ciclo con respecto a los AJ, lo que sugiere que este incremento en la separación de las piernas buscaría aumentar la base de sustentación en este grupo, para así aumentar la estabilidad durante la marcha ${ }^{15}$. Esto se relaciona a lo descrito por Schrager et al., quienes encontraron que producto del envejecimiento, el centro de masa corporal aumenta su excursión mediolateral, por lo que se hace necesario un cambio en la base de sustentación para disminuir el riesgo de caídas ${ }^{13}$.

En conclusión, las principales diferencias encontradas en el desplazamiento angular de la marcha a una velocidad controlada entre AJ y AM, se relacionan con cambios para mantener la estabilidad dinámica general en los AM. Una forma que éstos utilizarían para mantener la estabilidad sería a través de la reducción de rangos articulares en el tobillo con el objetivo de mejorar el posicionamiento de esta articulación para aumentar su base de sustentación (esto se logra en conjunto con un aumento de la abducción de cadera), lo cual es compensado con un aumento en los movimientos pélvicos anteriores, y una reducción en los laterales. De esta forma, en una marcha a una misma velocidad que los AJ, logran mantener los rangos articulares relacionados a la progresión en la marcha (plano sagital), buscando aumentar la estabilidad a través de cambios significativos de sus movimientos medio-laterales (plano frontal) ${ }^{26}$.

Finalmente, será importante determinar como la variabilidad cinemática observada en nuestros resultados y en estudios anteriores afectan en la estabilidad de la marcha, ya que aún existe discrepancia en este punto. Esto hace necesario que futuros estudios puedan relacionar pruebas biomecánicas con pruebas clínicas de tal forma que se pueda tener un mayor conocimiento de cómo cambia la estabilidad de los AM en tareas 
funcionales como la marcha durante el proceso de envejecimiento normal.

\section{Referencias}

1. Yancheng L, Kun L, Songhua Y, Ming S, Kevin L, Kuan Z. Gait phase varies over velocities. Gait Posture 2014; 39 (2): 756-60.

2. Yavuzer G. Three-dimensional quantitative gait analysis. Acta Orthop Traumatol Turc 2009; 43 (2): 94-101

3. Lord S, Sturnieks D. The physiology of falling: assessment and prevention strategies for older people. J Sci Med Sport 2005; 8: 35-42.

4. Bronstein AM, Brand T, Woollacott M, Nutt JG. Editors. Clinical disorders of balance, posture and gait. $2^{\text {nd }}$ ed. New York: Arnold; 2004

5. Horak F. Postural orientation and equilibrium: what do we need to know about neural control of balance to prevent falls? Age Ageing 2006; 35: Ii7-Ii11.

6. Goldspink G. Age-related loss of muscle mass and strength. J Aging Res 2011; 2012: 1-11.

7. Narici MV, Maffulli N. Sarcopenia: characteristics, mechanisms and functional significance. Br Med Bull 2010; $1-21$.

8. Pereira AF, Silva AJ, Costa AM, Monteiro AM, Bastos EM, Marques MC. Muscle Tissue Changes with Aging. Acta Med Port 2013; 26 (1): 51-5

9. Biolo G, Cederholm T, Muscaritoli M. Muscle contractile and metabolic dysfunction is a common feature of sarcopenia of aging and chronic diseases: From sarcopenic obesity to cachexia. Clin Nutr Res 2014; 33 (5): 737-48.

10. Shumway-Cook A, Woollacott M. Motor Control: Theory and practicalapplications. Philadelphia: Lippincott Williams \& Wilkins. 2001.

11. Kang HG, Dingwell JB. Effects of walking speed, strength and range of motion on gait stability in healthy older adults. J Biomech 2008; 41 (14): 2899-905.

12. Bergland A, Jarnlo GB, Laake K. Predictors of falls in the elderly bylocation. Aging Clin Exp Res 2003; 15 (1): 43-50.

13. Schrager MA, Kelly VE, Price R, Ferrucci L, Shumway-Cook A. The effects of age on medio-lateral stability during normal and narrow base walking. Gait Posture 2008; 28 (3): 466-71.

14. Hausdorff JM, Ríos DA, Edelberg HK. Gait variability and fall risk incommunity-living older adults: a 1-year prospective study. Arch Phys Med Rehabil 2001; 82 (8): 1050-56.

15. Helbostad JL, Moe-Nilssen R. The effect of gait speed on lateral balance control during walking inhealthy elderly. Gait Posture 2003; 18 (2): 27-36. [PubMed: 14654205]

16. Maki BE. Gait changes in older adults: predictors of falls or indicators offear? J Am Geriatr Soc 1997; 45 (3): 31320.

17. Lim YP. Muscle function during gait is invariant to age when walking speed is controlled. Gait Posture 2013; 38 : 253-9.

18. Shin S, Valentine R. Lower extremity muscle quality and gait variability in older adults. Age Ageing 2013; 41: 595-9.

19. Arnold JB, Mackintosh S, Jones S, Thewlis D. Differences in foot kinematics between young and older adults during walking. Gait Posture 2014; 39 (2): 689-94.

20. Legault-Moore D, Chester V, de Vries G. Multisegment foot kinematics during walking in younger and older adults. Journal of Clinical Medicine Research 2012; 4: 259.

21. Judge J, Davis R, Ounpuu S. Step length reductions in advanced age: the role of ankle and hip kinetics. Journals of Gerontology Series A Biological Sciences and Medical Sciences 1996; 51A: M303-12.

22. Dingwell, J.B., Cusumano, J.P., Sternad, D., Cavanagh, P.R. Local dynamicstability versus kinematic variability of continuous overground and treadmillwalking. ASME Journal of Biomechanical Engineering. 2001; 123 (1): 27-32.

23. Dingwell JB, Marín LC. Kinematic variability and local dynamic stability ofupper body motions when walking at different speeds. J Biomech Eng 2006; 39 (3): 444-52.

24. Perry J. Gait Analysis. First ed. Thorofare: SLACK Incorporated. 1991.

25. Crosbie J. Synchrony of pelvic and hip motion during walking. Gait Posture 1997; 5: 237-48

26. Sparrow WA, Tirosh O. Gait termination: a review of experimental methods and the effects of ageing and gait pathologies. Gait Posture 2005; 22 (4): 362-71. 